\title{
Design and Implementation of a Cost Effective Quartz Crystal Microbalance System for Monitoring Small Changes on any Surface
}

\author{
S. TURKDOGAN
}

\begin{abstract}
Quartz crystal microbalance systems are widely used in different fields from biology to electronic. Those devices are very sensitive to mass changes on the piezoelectric crystal surface in nano gram scale and therefore can be used to detect even a single virus on surface of any substance. Although they are very practical and versatile, they are sold with very high prices and thus hindering the usage for someone with tight research budget. In this work, we try to find a cost effective solution and bring this device into the price range on two to three orders of magnitude lower than the commercial counterparts. For that purpose, we develop a cost effective quartz crystal microbalance system using a cost effective microcontroller and a typical oscillator circuit and the final product can be used in different fields to detect the mass change on any surface caused by different reactions or effects.
\end{abstract}

Index Terms - Piezoelectric, QCM (Quartz Crystal Microbalance), Sensors, Transducers, Thickness/Gas Monitor.

\section{INTRODUCTION}

$\mathrm{P}$ IEZOELECTRIC EFFECT produced by piezoelectric crystals sandwiched between two metal electrodes is an essential phenomena used in electronic applications. When we compress a piezoelectric material such as Quartz it produces an electric charge. However, it works in other way as well and when the electric current is flown it changes the shape slightly and creates a mechanical vibration [1]. In electronics, we utilize the first effect, but latter effect can also be utilized in some applications. Besides producing clock pulses for electronic circuits, piezoelectric crystal can also be used as a mass sensor that is known as Quartz Crystal Microbalance (QCM) [2-7]. They are of great interest for a wide variety of measurements in many fields as they are able to work in different operating conditions in gas or liquid mediums [1-9]. In addition, they offer high sensitivity, high stability, low power consumption and easy operation at room temperature.

SUNAY TURKDOGAN, is with Department of Electrical and Electronics Engineering University of Yalova, Yalova, Turkey, (e-mail: sunayturkdogan@hotmail.com).

iD https://orcid.org/0000-0002-9690-325X

Manuscript received February 22, 2019; accepted May 10, 2019. DOI: $\underline{10.17694 / \text { bajece. } 530796}$
QCM sensors are well-suited for the sensor arrays used in environmental, biomedical and chemical applications. For example, in a recent study [10] QCM was utilized to detect the water vapor adsorption level (humid) by nanomaterials in different morphologies. As the morphology changes from thin film to nanowire the surface area significantly increase and therefore more water molecules in gas form can adsorb on their surface which eventually casue to shift resonance frequency of QCM crystal more due to the mass uptake. Other studies also utilize QCM to detect any other gas molecules. In addition to those, QCM can be also used for the following applications. We can measure the real time addition of molecular layers, swelling and desorption rates, rigidity, thickness and water contents, binding events, induced changes in viscoelasticity and thickness, and cellular attachments in parallel with microscopy on any surface.

Our main purpose of this study is to bring such a versatile QCM device into the life with substantially low cost and comparable device performance and make everyone utilize it. This work would be a good reference for someone in a tight budget because the developed system will be on par with the commercial counterparts except with a massive lower cost.

\section{WORKING PRINCIPLE OF QCM}

QCM is a widely used acoustic wave sensor to figure out the mass change on any surface of a substance as a function of the change on the quartz crystal's resonance frequency. We can calculate the mass change on thin and uniform films using Sauerbrey equation [10]:

$$
\Delta \mathrm{m}=-\frac{A \sqrt{\mu \rho}}{2 f_{o}^{2}} \Delta \mathrm{f}
$$

where $\mathrm{A}$ is the active electrode layer area on the crystal, $\mu$ the shear modulus of quartz, $\rho$ density of the crystal, fo resonance frequency of the crystal, and $\Delta \mathrm{f}$ frequency shift from its fundamental resonance frequency.

Our QCM device utilizes an AT-cut quartz crystal with resonance frequency of $5 \mathrm{MHz}$. AT-cut crystals widely used by the industry are made from $\mathrm{Y}$ bar at $35^{\circ}$ rotation (lower right of Fig. 1) and those provide a stable performance over a wide temperature range $\left(-55^{\circ} \mathrm{C}-125^{\circ} \mathrm{C}\right)$. In our device, the Shear Modulus $(\mu)$ and density $(\rho)$ of the crystal are $2.947 \times 1011$ $\mathrm{g} / \mathrm{cm}^{2} \mathrm{~s}^{2}$ and $2.684 \mathrm{~g} / \mathrm{cm}^{3}$, respectively. According to Sauerbrey equation (1), the change of $\pm 1 \mathrm{~Hz}$ of the resonance frequency corresponds to $5.02 \mathrm{ng}$ of materials desorbed/adsorbed on the $0.282735 \mathrm{~cm}^{2}$ active crystal surface. It is important to note that 
a QCM sensor with low resonance frequency should be heavy in order to eliminate the vibrational effects caused by the environment. However, we will utilize a crystal oscillating in a frequency greater than $5 \mathrm{MHz}$ and will automatically prevent the occurrence of the aforementioned problem because in nature there is no such vibrations. This will make our QCM system very lightweight.

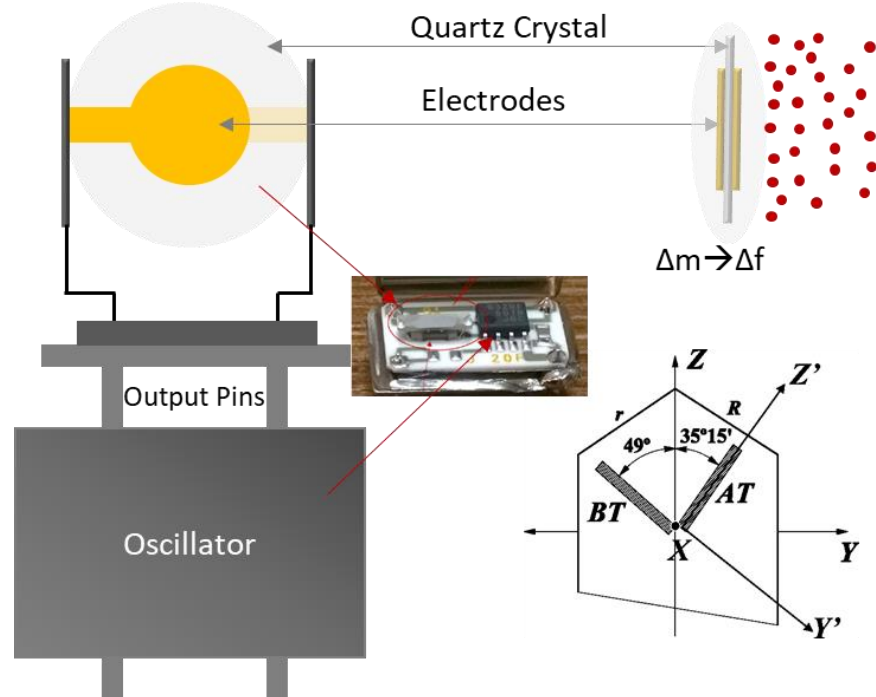

Fig.1. Schematic view of the quartz crystal from side and cross sectional views. Lower right is the demonstration of AT-cut crystal orientation.

\section{RESULTS AND DISCUSSION}

\section{A. Design and simulation of a $Q C M$}

The idea behind a QCM circuitry is very simple and as follows. The circuit basically consists of a crystal oscillator and the signal produced by this oscillator is detected by a microcontroller and measures the oscillation frequency of the corresponding signal. As previously mentioned this signal is proportional to the mass change over the active electrodes of the piezoelectric crystal. When the crystal adsorbs some specious, negative frequency shift occurs in the oscillation frequency while this turns to be opposite in case of the mass desorption. Various crystal oscillator circuits can be designed, but for the sake of easiness, we can exploit a cost effective crystal oscillator in a single package with potentially different fundamental oscillation frequencies. It is important to note that $1 \mathrm{~Hz}$ frequency shift corresponds to a lower mass change as the fundamental oscillation frequency $\left(\mathrm{f}_{\mathrm{o}}\right)$ gets higher because in Eq.(1), mass change is inversely proportional to the square of $f_{o}$. It means that as $f_{o}$ increases we get more sensitive measurements. In this research, we utilized TCO-711A oscillator, which can be used between $0.25 \mathrm{MHz}$ and $100 \mathrm{MHz}$ [12]. This oscillator has hermetically sealed package to prevent any effect caused by the environment; however, we cut the package in order to reach two pins of the crystal connections (see Figure $3 \mathrm{~b}$ ) and those connections could be extended for our convenience in the measurement procedure. Figure 1 shows the cut package and crystal can be clearly seen. This crystal can be replaced by any other crystal operating in the aforementioned frequency range and this determines the oscillation frequency of the system. By changing the crystal pointed in Fig. 1 we can use the oscillator in the indicated range. This circuit uses the second harmonic of the crystals meaning that whatever rated crystal we use we can get half of the rated oscillation frequency. For example, if we replace the crystal with $10 \mathrm{MHz}$ crystal we can get $5 \mathrm{MHz}$ signal out of TCO-711A. Since we are able to replace the quartz crystal, it gives a chance to do disposable tests. It is recommended that only crystals rather than TCO-711A oscillator (top left component seen in Figure $3 \mathrm{~b}$ ) can be bought and by cutting the package quartz crystals can be taken and face to the environment with a much lower price.

By replacing the original crystal in TCO-711A with HEC $10 \mathrm{MHz}$ crystal we test our oscillator and the signal produced by the modified oscillator was analyzed using two channel oscilloscope. Figure 3a shows the signal oscillating around $5 \mathrm{Mhz}$. We will design a circuit to detect this signal and project the result in any serial monitor.

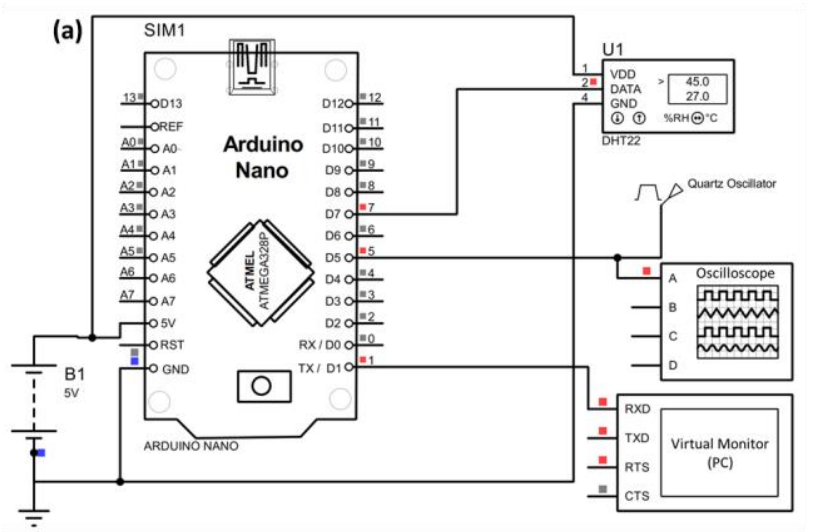

(b)

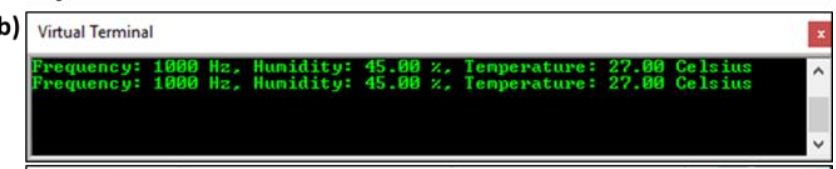

(c)

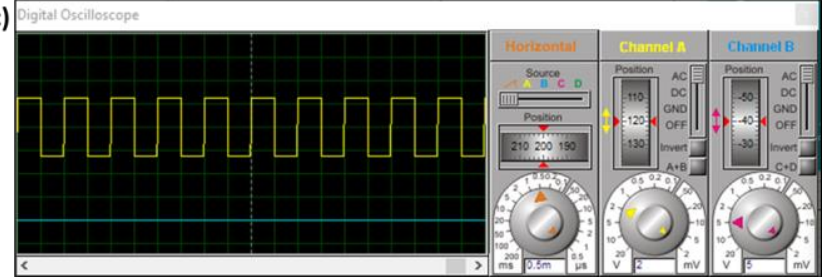

Fig.2. (a) Circuit diagram of the designed QCM system. (b) Simulation results obtained from the virtual terminal. (c) Oscilloscope view of the signal used in simulation to test our QCM device.

Before implementing the designed circuit, a simulation using Proteus 8.0 was done for the system seen in Fig. 2a. In this simulation, a square wave signal with $50 \%$ duty cycle was used as the signal produced by crystal oscillator and a model of DHT22 was used to replicate the humidity/temperature sensor. Arduino Nano microcontroller working $16 \mathrm{MHz}$ clock speed was utilized to evaluate the data gathered by sensors and oscillator and project the results on a virtual terminal used as a serial monitor. It is important to note that test signal was $1 \mathrm{kHz}$ as seen in Fig.2c. The reason for that the signal is disturbed as we increase the frequency to $\mathrm{MHz}$ ranges; however, the real device is tested under $5 \mathrm{MHz}$ as we will see later. The program we compiled to make this circuit operational was written in Arduino IDE and seen in Appendix section. In the simulation, 
we get the temperature, humidity and frequency values and monitored those in virtual terminal as seen in Fig. $2 b$.

\section{B. Prototype circuit and experimental results}

After a successful operation of our device in Proteus we implemented our circuit on a breadboard as seen in Fig. $3 b$. Before testing, the capability of frequency range that our device can run, $50 \%$ duty cycle signals with a wide range of frequency from $1 \mathrm{kHz}$ to $10 \mathrm{MHz}$ was tested. Since our microcontroller work with $16 \mathrm{MHz}$ clock speed and we are limited with the frequency up to $8 \mathrm{MHz}$ (half of clock speed) by using "FreqCount" command which can be found in [13]. By employing more advanced microcontrollers such as Teensy 3.0, Teensy 3.5 etc. working with high clock speed we can measure the oscillation frequency greater than $8 \mathrm{MHz}$. After testing the capability of frequency read, we build up our system on the breadboard and read the detected values on the PC screen. Although our aim is to measure the oscillating frequency of the crystal, we embedded a temperature and humidity sensor because correlation of humid, temperature and frequency shift is required to make a meaningful comment in many applications.
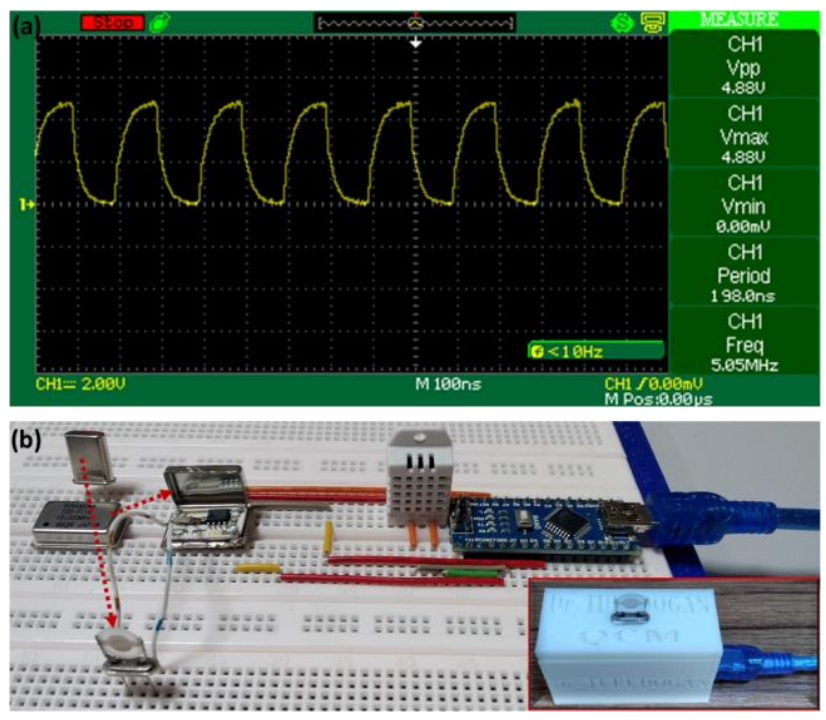

(c)
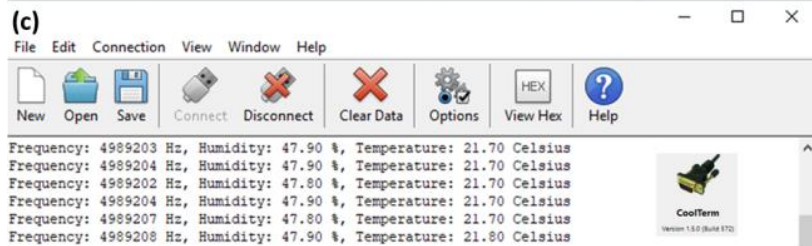

Freglency: 4989207 Hz, Humidity: 47.80 I, Temperature: 21.70 Celsius

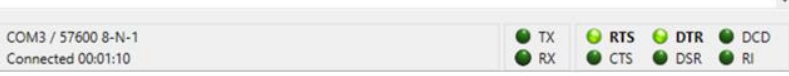

Fig.3. (a) Real oscillating signal captured by 2 channel oscilloscope showing $5 \mathrm{MHz}$ oscillation frequency. (b) Real color image of the system built on a breadboard. Inset: 3D printed casing for the circuit to make our device more compact. (c) Data on the screen was sent by the QCM device and projected on the "CoolTerm" screen via USB connection between PC and circuit.

As seen in Fig.3c, the received data is projected on a free program called "CoolTerm". Our microcontroller sends the data with 57600 baud rate and the program is set to that value (lower left of Fig.3c) to capture them. The reason we used this program is because the data seen on the screen can simultaneously be saved into the PC and the saved data can be later used for data processing. In other words, we can utilize this program as a cost effective data logger. By $3 \mathrm{D}$ printing a case for our system as seen in Fig. 3c, we can make everything compact and implement tests in different environments easily.

As a proof of concept, we test our device in a hermetically sealed humidity test chamber and the results are shown in Fig. 4. The data was obtained in about twelve minutes and with some intervals humidity was altered by flowing a humid air into the chamber. The result shows that the oscillation frequency shifts to lower values as we increase the humidity and the oscillation frequency increases as the humidity gets to the original ambient value. This is as we expected because when the water molecules adsorbed by the electrodes of the crystal, mass increases and this shifts the oscillation to lower frequency values. In addition, the frequency increases as the adsorbed molecules starts to desorb from the surface. It is worth to note that the frequency change is not very high in this case because the electrode surface is very smooth and there is no enough surface area to be adsorbed on. If we coat the electrodes with some nanomaterials having very high surface area, the change in the oscillation frequency becomes more intense. By this way, we can utilize a QCM to detect any phenomenon occurring in gasses or liquid environments.

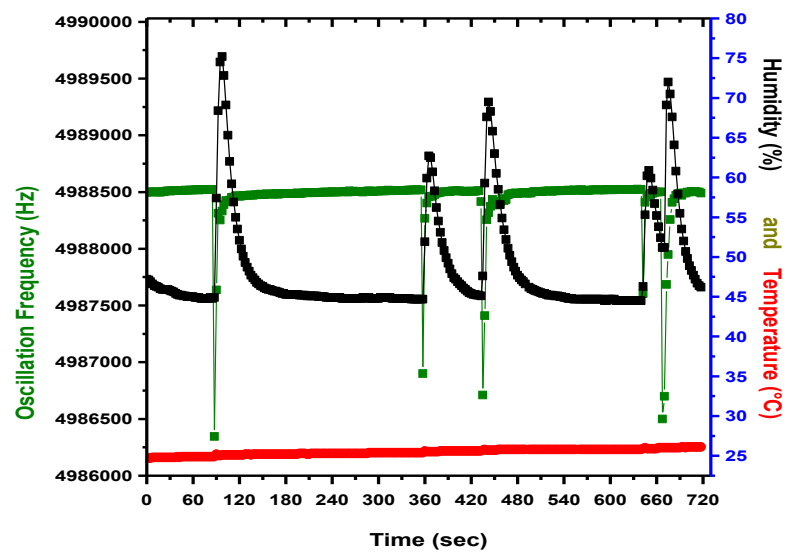

Fig.4. A graph showing the experimental data gathered via our device in a given time.

\section{CONCLUSION AND FUTURE WORK}

In conclusion, we have developed, simulated and implemented a simple but versatile QCM device employing cost effective components and integrated circuits. The results show that it works very satisfactorily. Although our design is very easy to implement it can substitute a very expensive and more complex QCM device and realize the sensitive analysis in different fields with low-cost. This design is believed to be widely speeded all over the world and will be utilized for sensing variety of gases, depositions, desorption and etc. and contribute the development of science. As a future work, we are planning to integrate an OLED/LCD display, real time 
clock and SD card writer/reader and by this way, we can carry a complete QCM test system in our pocket. This circuit will show the frequency shift, mass change, temperature and humidity information as a function of time on the OLED/LCD screen and can save all these data into a micro SD card to be used in data processing.

\section{ACKNOWLEDGMENT}

This work was supported by the Scientific Research Fund of Yalova University under the project number 2017/AP/140.

\section{REFERENCES}

[1] A. Alassi, M. Benammar, D. Brett, "Quartz crystal microbalance electronic interfacing systems: A review." Sensors, Vol.17, 12, (2017), pp. 2799.

[2] K.N. Chappanda, O. Shekhah, O. Yassine, S.P. Patole, M. Eddaoudi, K.N. Salama, "The quest for highly sensitive QCM humidity sensors: The coating of CNT/MOF composite sensing films as case study" Sensors and Actuators B: Chemical, Vol.257, (2018), pp.609-619.

[3] X. Cha, F. Yu, Y. Fan, J. Chen, L. Wang, Q. Xiang, J. Xu, "Superhydrophilic $\mathrm{ZnO}$ nanoneedle array: Controllable in situ growth on QCM transducer and enhanced humidity sensing properties and mechanism" Sensors and Actuators B: Chemical, Vol.263, (2018), pp.436-444.

[4] A. Erol, S. Okur, N. Yağmurcukardeş, M.Ç. Arıkan, "Humidity-sensing properties of a $\mathrm{ZnO}$ nanowire film as measured with a QCM" Sensors and Actuators B: Chemical, Vol.152, (2011), pp.115-120.

[5] F. Pascal-Delannoy, B. Sorli, A. Boyer, "Quartz crystal microbalance (QCM) used as humidity sensor" Sensors and Actuators A: Physical, Vol.84, (2000), pp.285-291.

[6] B. Ding, J. Kim, Y. Miyazaki, S. Shiratori, "Electrospun nanofibrous membranes coated quartz crystal microbalance as gas sensor for NH3 detection" Sensors and Actuators B: Chemical, Vol.101, 3, (2004), pp.373-380.

[7] I. A. Koshets, Z. I. Kazantseva, Y. M. Shirshov, S. A. Cherenok, V. I. Kalchenko, "Calixarene films as sensitive coatings for QCM-based gas sensors" Sensors and Actuators B: Chemical, Vol.106, 1, (2005), pp.177-181.

[8] M. M. Kamel, M. K. El Nimr, S. T. Assar, A. S. Atlam, "Design of a Simple Low-Cost Quartz Crystal Microbalance System" Instrumentation Science \& Technology, Vol.41, 5, (2013), pp.473-489.

[9] E. S. Muckley, C. Anazagasty, C. B. Jacobs, T. Hianik, I. N. Ivanov, "Low-cost scalable quartz crystal microbalance array for environmental sensing" In Organic Sensors and Bioelectronics IX International Society for Optics and Photonics, Vol.9944, (2016), pp.99440Y.

[10] G. Sauerbrey, "The use of quartz crystal oscillators for weighing thin layers and for microweighing applications" Zeitschrift für Physik, Vol.155, 3, (1959), pp.206-222.

[11] Turkdogan, S. J Mater Sci: Mater Electron (2019). https://doi.org/10.1007/s10854-019-01384-z

[12] "Crystal Oscillator." TOYOCOM. Accessed February 21, 2019. http://eshop-bg.com/PDFs/CrystalOscillators/TOYOCOM_TCO711A.pdf

[13] "FreqCount Library." PJRC. Accessed February 21, 2019. https://www.pjrc.com/teensy/td_libs_FreqCount.html.

\section{BIOGRAPHY}

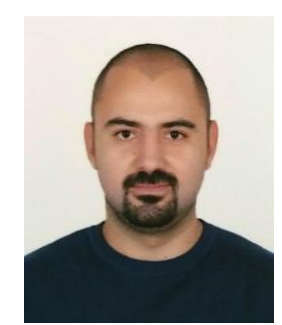

SUNAY TURKDOGAN received his B.Sc. degree in Electronic Engineering from Uludag University, Turkey in 2007 and he got both his M.S. and Ph.D. degrees in Electrical Engineering from Arizona State University, US in 2012 and 2015, respectively. Currently, he is an Associate Professor in the Department of Electrical and Electronics Engineering at University of
Yalova, Turkey and his research mainly focuses on nanotechnology, nanophotonics, nanomaterial growth and characterization, optical devices, sensors, solar cells and photovoltaic systems. 


\section{APPENDIX}

//Libraries

\#include <DHT.h>

\#include <FreqCount.h>

//Constants

\#define DHTPIN 2 // What pin we're connected to

\#define DHTTYPE DHT22 // DHT 22 type of sensor is used

DHT dht(DHTPIN, DHTTYPE); //// Initialize DHT22 sensor

//Variables

float hum; //Stores humidity value

float temp; //Stores temperature value

void $\operatorname{setup}()$

\{

Serial.begin(57600);

FreqCount.begin(1000);

dht.begin();

\}

void $\operatorname{loop}()$

\{

if (FreqCount.available()) \{

unsigned long count $=$ FreqCount $\cdot \operatorname{read}()$;

//Print frequency value to serial monitor

Serial.print("Frequency: ");

Serial.print(count);

Serial.print(" Hz, Humidity: ");

\}

//Read data and store it to variables hum and temp

hum $=$ dht.readHumidity () ;

temp $=$ dht.readTemperature () ;

//Print temp and humidity values to serial monitor

Serial.print(hum);

Serial.print(" \%, Temperature: ");

Serial.print(temp);

Serial.println(" Celsius");

delay(2000); //Delay 2 sec. Sampling rate of DHT22 $0.5 \mathrm{~Hz}$

\} 